\title{
Role of Antioxidants in the Treatment of Male Infertility
}

\author{
Khaled Gadallah* \\ Department of Urology, Al-Azhar University, Cairo, Egypt \\ *Corresponding author: Khaled Gadallah, Department of Urology, Al-Azhar University, Cairo, Egypt
}

Submission: November 13, 2017; Published: January 17, 2018

\begin{abstract}
Stress is defined as a general body response to initially threatening external or internal demands, involving the mobilization of physiological and psychological resources to deal with them. Recently, oxidative stress has become the focus of interest as a potential cause of male infertility. Normally, equilibrium exists between reactive oxygen species (ROS) production and antioxidant scavenging activities in the male reproductive organs.

Non-hormonal medical treatment has been proposed for patients with idiopathic or non-curable oligo-astheno-terato-zoospermia and for normo-zoospermic infertile patients. Anti-inflammatory, fibrinolytic \& antioxidant compounds, oligo elements and vitamin supplementation may be prescribed. Infection, inflammation and/or increased oxidative stress often require a specific treatment with antibiotics, anti-inflammatory drugs and/or antioxidants. The ascorbic acid is a known antioxidant present in the testis with the precise role of protecting the male reproductive organs from the oxidative damage. It also contributes to the support of spermatogenesis at least in part through its capacity to maintain antioxidant in an active state. Combined therapies can contribute to improve sperm quality.
\end{abstract}

\section{Introduction}

Males are found to be solely responsible for $20-30 \%$ of infertility cases (at least 30 million men worldwide) and contribute to $50 \%$ of cases overall (Agarwal et al., 2015) [1]. Oxidative stress has been identified as a major mediator in various etiologies of male infertility. Treatments of oxidative stress, including oral antioxidants and varicocelectomy, have been studied widely in patients with varicocele-associated male subfertility and unexplained male infertility. Current assays for seminal oxidative stress can measure reactive oxygen species (ROS) directly or indirectly [2].

The sources of ROS in semen are both intrinsic and extrinsic. Activated leukocytes (mainly polymorph nuclear leukocytes \& macrophages) resulting from inflammation and infection are significant intrinsic producers of ROS in semen [3].

Immature spermatozoa with abnormal head morphology and cytoplasmic retention are another important source [4]. Damaged, deficient or abnormal spermatozoa as a result of impaired spermatogenesis can yield excessive ROS as well [5].

Sertoli cells in semen have also been shown to possess the ability to produce ROS [6]. Other intrinsic etiologies include varicocele (higher grade is associated with greater amounts of ROS production), cryptorchidism, testicular torsion and ageing [7].

Extrinsic sources such as cigarette smoking [8], alcohol consumption [9], exposure to radiation [1] and other environmental toxins have been associated with elevated testicular and/or seminal ROS levels. Common sources of ROS in semen and their adverse effects are illustrated in (Figure 1) [7].

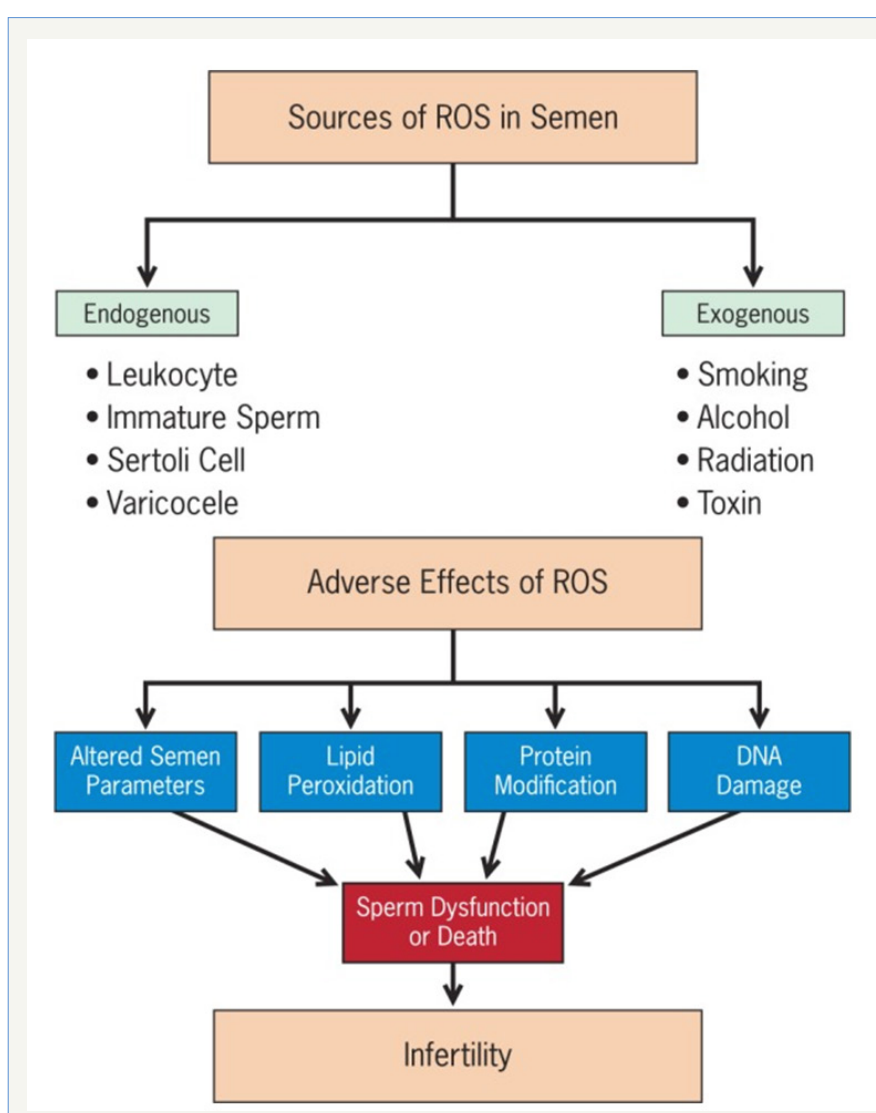

Figure 1: Common sources of excessive reactive oxygen species (ROS) in semen and their deleterious effects [7]. 
ROS levels can affect sperm structural and functional integrity including motility, morphology, count and viability, thereby making it one of the important etiologies of male factor infertility. High ROS concentrations in infertile men have been associated with DNA fragmentation and poor chromatin packing. Sperm DNA damage may decrease fertilization rates, reduce implantation, impair embryonic development and increase miscarriage/pregnancy loss and the potential for birth defects [10]. ROS affect mitochondrial or nuclear DNA of sperm at an amino acid or molecular level in the form of base modification (especially guanine), attacking the phosphor-di-ester backbones and producing base-free sites, point mutations, polymorphisms, deletions, translocations, strand breaks and chromatin cross-links, frame shifts and even rearranging chromosomes [11].

Sperm chromatin has a highly condensed and organized structure that helps to protect it from oxidative damage, but when compaction is poor and chromatin protamination is incomplete, sperm DNA is more vulnerable to ROS. DNA damage is a contributory factor to apoptosis [12] and in cases of more severe damage of spermatozoa, apoptosis results in low sperm counts characteristic of idiopathic male factor infertility [13].

\section{Alleviation of oxidative stress and potential treatment strategies}

Treatment of oxidative stress-associated male infertility has been studied widely in patients with varicocele-associated subfertility and unexplained infertility. In these patients, treatment strategies such as surgical varicocele repair (varicocelectomy) and oral antioxidant therapy have been used.

\section{Oral antioxidant therapy}

In spite of the conflicting data, many urologists prescribe oral antioxidant therapy for men with clinical subfertility with or without varicocele [14]. The rationale for recommending oral antioxidant therapy is based on the premise that seminal oxidative stress is due in part to a deficiency in seminal antioxidants and the lack of serious side effects related to antioxidant therapy. Ideally, an oral antioxidant should reach high concentrations in the reproductive tract and restore vital elements important for spermatogenesis. Additionally, the antioxidant supplement should augment the scavenging capacity of seminal plasma and reduce levels of seminal ROS [15].

Oral treatment with antioxidants such as vitamins E, C, A, B complex, coenzyme Q10 (CoQ10), ubiquinol, glutathione, l-carnitine, lactoferrin, $\beta$-carotene, lycopene, pantothenic acid, $\alpha$-lipoic acid, $\mathrm{N}$-acetyl-cysteine, selenium, zinc, copper or supplements containing a combination of these antioxidants have been used with success to varying degrees [16].

In infertile men, oral antioxidant therapy with Carni-Q-Nol (440mg l-carnitine fumarate $+30 \mathrm{mg}$ ubiquinol+ $75 \mathrm{IU}$ vitamin $\mathrm{E}$ $+12 \mathrm{mg}$ vitamin C) softules twice or thrice daily decreased sperm pathology after 3 months, improved sperm density after 3 and 6 months, increased (ubiquinone + ubiquinol) \& $\alpha$-tocopherol and decreased oxidative stress levels. Apart from improving sperm parameters, the supplementary therapy with Carni-Q-Nol resulted in a $45 \%$ pregnancy rate [17].

Moreover, antioxidant supplements are not free from potential side effects "antioxidant paradox". Further placebo-controlled, dietary-controlled, double-blind, randomized-controlled, prospective studies with standardized supplement regimens are needed to elucidate the role of antioxidant therapy in the treatment of oxidative stress and management of male infertility [7].

\section{Antioxidants}

Protective antioxidant system comprehends enzymatic factors (superoxide dismutase (SOD), catalase and glutathione peroxidase (GPX), non-enzymatic factors and low molecular weight compounds (glutathione, N-acetyl-cysteine (NAC), vitamins A, E \& C, coenzyme Q10 (CoQ10), carnitines, myoinositol (MYO), lycopene, astaxanthin, Serenoa repens, etc.), and micronutrients (Selenium, Zinc, \& Copper) which interact each other to ensure an optimal protection against the oxidative stress. A deficiency of one of them may result in a decrease of total plasma antioxidant capacity [18]. The main antioxidants will be discussed below.

\section{Superoxide dismutases}

SODs are metalloenzymes that convert superoxide to hydrogen peroxide (H2O2) and comprehend two intracellular and one extracellular forms. The intracellular form contains copper and zinc in the active center (CuZnSOD). It is encoded by the SOD1 gene and it is mainly localized in the cytoplasm. The other intracellular form is manganese SOD (MnSOD) that acts in the mitochondrial matrix [18]. Its gene, SOD2, is inducible under various inflammatory conditions and the nuclear factor kappa B (NF-kB) appears to be the main factor responsible for its induction. Homozygous SOD2 genedeficient mice have severe cardiovascular damage and die soon after birth. No abnormality in the genital tract has been reported in these mice. On the contrary, transgenic male mice that express higher levels of MnSOD are infertile. Since SOD only dismutates superoxide anion into hydrogen peroxide, the resulting H2O2 may also cause a toxic effect in testicular cells [19].

The extracellular form of SOD (ECSOD), encoded from SOD3 gene, can be present in a free form or can be connected to the surface polysaccharides. It is structurally similar to the MnSOD, but it has zinc and copper in the active center. It has been shown that erectile function is improved by transferring the SOD3 gene to the penis in aged rats. Scavenging superoxide increases nitric oxide (NO) half-life that results in increased cGMP levels [19].

The main active isoenzymes in the seminal plasma are CuZnSOD (75\%) and ECSOD (25\%) which probably originate from the prostate [20]. SODs have been observed to play a role in the protection of testicular cells against heat stress-induced apoptosis both in vivo and in vitro [21]. Moreover, SOD activity has been lower in infertile patients than in fertile controls and correlates positively with sperm motility and morphology [22]. 
Some in-vitro studies suggest that SODs may play a role in improving sperm parameters in thawed semen samples. In fact, supplementation with manganese (III) mesotetrakis (N-ethylpyridinium-2-yl) porphyrin chloride (MnTE), a permeable SOD mimetic agent, seems to improve total motility, membrane integrity, and viability of goat semen samples after thawing, the acrosomal integrity and the blastocyst formation rate [23]. However, since MnTE supplementation protects spermatozoa from superoxide but not from H2O2, the degree of sperm parameters improvement is higher when also catalase is simultaneously added [24]. There are evidences for positive effects of the treatment with SODs also on human spermatozoa. In fact, the supplementation of exogenous SODs at the dose of $400 \mathrm{U} / \mathrm{mL}$ to the human sperm suspension prevented the loss of motility and the increase of MDA concentration, thus showing a significant role of SODs for human sperm motility. Actually, SOD is included in some over-the-counter products that contain also other anti-antioxidants (e.g., D-chiroinositol, zinc, and folic acid), recommended for the treatment of male infertility. There is a lack of studies investigating the effects of SOD oral supplementation on human sperm. Hence, there is not agreement about the dosage. However, the most common therapeutic scheme suggested consists of an oral dosage of $150 \mathrm{UI}$ a day for at least three months [25].

\section{Catalase}

Catalase is a heme enzyme with a centrally located iron atom. It catalyzes the decomposition of $\mathrm{H}_{2} \mathrm{O}_{2}$ into molecular oxygen $\left(\mathrm{O}_{2}\right)$ and water $\left(\mathrm{H}_{2} \mathrm{O}\right)$. Its presence has been shown in the seminal plasma and spermatozoa of both humans and rats. The extracellular enzyme is produced by the prostate.

Under physiological conditions, catalase plays a role in the nitric oxide-induced sperm capacitation [18]. Its activity has been associated with low sperm quality [26]. In fact, H2O2, which increases in case of catalase deficiency or reduced activity, plays a substantial role on sperm motility due to the decrease in membrane fluidity that is important for sperm-oocyte fusion [27]. It is not commercially available.

\section{Glutathione peroxidase}

GPXs reduce hydrogen peroxide and organic peroxides, including phospholipids, using the reduced form of glutathione (GSH) as an electron donor [28]. GPXs protect sperm DNA from oxidative damage, playing a role in the chromatin condensation and mainly in the mitochondrial matrix [18]. GPX-defective expression in human spermatozoa has been reported to be associated with OAT [29].

GPXs are divided into two groups according to the presence of seleno-cysteine in the enzyme isoform. Since, selenium deficiency has been associated with male infertility, selenium-containing GPXs are suspected to be a candidate for the defective molecule. At least four isoenzymes belong to the selenium-containing GPX in mammals.

GPX1 is a cytosolic isoform, widely distributed in tissues and, like other antioxidant enzymes, and prevents apoptosis induced by oxidative stress and other stimuli. GPX1 knockout mice do not show reproductive abnormalities. GPX2 and GPX3 are gastrointestinal and plasma isoforms, respectively.

GPX4 is highly expressed in testis and represents about the 50\% of the capsule material, which embeds the helix of mitochondria in the midpiece of spermatozoa. GPX4 gene knockout mice show premature embryonal death. The GPX4 gene encodes also for a protein that has a high sequence identity to GPX4 except for the $\mathrm{N}$-terminal region. This protein is specifically present in sperm nuclei and is considered to act as protamine thiol peroxidase.

GPX5 is a non-selenium enzyme. It is exclusively expressed in the epididymis and secreted in the caput and caudal epididymal lumen. It represents the $6 \%$ of the secretory epididymal proteins. However, the activity of non-selenium dependent GPX in low and, hence, its contribution as a GSH-dependent peroxide scavenger is ambiguous [19]. However, neither catalase nor GPXs are currently used for the treatment of male infertility.

\section{Glutathione}

Glutathione is a sulfur-containing tripeptide present in both a reduced (GSH) and an oxidative (GSSG) states. GSH participates in preserving the intracellular milieu in a reduced state and, in addition, it is an electron donator to GPX. GSH displays its antioxidant activity by the reconstruction of thiol groups $(-\mathrm{SH})$ in proteins and preventing cell membrane from lipid oxidation [18].

GSH levels are maintained through two metabolic pathways: one is the de novo synthesis from Cys, Glu, and Gly that is catalyzed by the $\gamma$-glutamyl-cysteine synthetase (GCS) and glutathione synthetase; the other is its recycling by glutathione reductase, using NADPH as an electron donor. Glutathione is pumped out when it is oxidized or forms conjugates with cytotoxic compounds, including xenobiotical chemicals. Plasma glutathione is hydrolyzed $\gamma$-glutamyltranspeptidase (GGT), localized at the cellular surface as a membrane protein, into its consisting amino acids that are then taken up by the specific transporter and reused by cells [19].

In animals, GSH therapy affects positively sperm quality. It has a crucial role in increasing sperm motility and fertilization in bulls with asthenozoospermia due to varicocele and in rabbits with dispermy caused by cryptorchidism [30].

Glutathione reduction in human seminal plasma leads to instability of the midpiece of the spermatozoa, resulting in a motility disorder [31]. Some authors suggested that its supplementation plays a therapeutic role in some andrological diseases, particularly during inflammation. Accordingly, its supplementation in infertile men with unilateral varicocele or inflammation of the urogenital tract (two conditions in which radical oxygen species or another toxic compound production plays a pathogenetic function) leads to a significant improvement of sperm parameters, such as concentration, motility and morphology [32]. These effects on spermatozoa can be partially reversed in case of not too severe structural cell membrane damage [31], a prominent protective role of GSH on the lipid components of the cell membrane [33]. 
Glutathione is given at the dosage of $600 \mathrm{mg} /$ day intramuscularly for 2-3 months. Hence, glutathione is seldom prescribed for male infertility. However, because of the route of administration, the compliance is low and, currently, GSH is hardly used for the treatment of male infertility.

\section{$\mathrm{N}$-Acetyl-Cysteine (NAC)}

NAC is a glutathione precursor. NAC is effective in metal chelation and it seems to improve sperm motility and to prevent sperm DNA oxidative damage [18]. In an animal model, it is able to improve sperm parameters and seminal vesicles weight, previously altered by treatment with As203 [34]. Furthermore, it significantly improves seminal fluid volume and viscosity and increase sperm motility in humans [35]. In women suffering from polycystic ovary syndrome, NAC (administered at the doses of $1500 \mathrm{mg} /$ day) has been shown to ameliorate oocyte and embryo quality, representing an alternative to metformin prescription [36]. Such findings support the therapeutic use of this compound for the treatment of human infertility.

The most common oral dosage used is $600 \mathrm{mg} /$ day. NAC is commercialized in combination with other antioxidants. Its administration is suggested for at least three months.

\section{Vitamin A}

Carotenoids are a group of fat-soluble organic compounds found mainly in yellow, red, orange and pink vegetables. These carotenoids are precursor of vitamin A. Retinal is formed from them in the gastrointestinal tract, before being converted to retinol, the most important component of vitamin A.

Carotenoids are natural antioxidants that protect cell membrane integrity, regulate epithelial cell proliferation, and are involved in the regulation of spermatogenesis [18]. Moreover, in rats retinoids have various effects on fetal and neonatal Sertoli, germ and Leydig cell [37]. Carotenoid deficiency in the diet can lead to a reduction in sperm motility [18]. Studies conducted on bulls have shown that retinol might stabilize sperm acrosomal membrane when oxidative stress increases because of the high temperature [38]. In humans, lower serum retinol concentration has been correlated to a worse sperm quality [39]. Hence, vitamin A administration could be a therapeutic choice for the treatment of human male infertility. Not many studies have evaluated the effects of vitamin A on human sperm parameters. Currently, it is seldom added to the over-thecounter preparation for male infertility treatment. However it should be strengthening the concept that vitamin A has been shown to be toxic and may be teratogenic in higher dose [40].

\section{Vitamin C (Ascorbic Acid)}

Vitamin C has a 10-fold higher concentration in seminal plasma than in the serum [41]. It has a more powerful antioxidant action when peroxyradicals are present in the aqueous phase [42] than in the lipid membrane [43]. In mice, at a concentration equivalent to the human therapeutic dose $(10 \mathrm{mg} / \mathrm{Kg})$, it is able to reduce MDA concentration, increasing sperm count and the proportion of normal sperm population [44].
In humans, the seminal acid ascorbic levels correlate positively with the percentage of morphologically normal spermatozoa [45] and negatively with DNA fragmentation index [46]. This finding supports the therapeutic use of vitamin C in infertile males. At concentrations lower than $1000 \mu \mathrm{mol} / \mathrm{L}$, vitamin $\mathrm{C}$ is an antioxidant; at higher concentrations it acts as a prooxidant agent [47]. The dose of $1 \mathrm{~g} /$ day provides a 2.2 -fold increase in plasma acid ascorbic concentrations [48]. The majority of the studies reported in literature investigating the effect of vitamin $\mathrm{C}$ administration on sperm quality refer to this orally administered dosage [49]. Accordingly, $1 \mathrm{~g}$ daily vitamin $\mathrm{C}$ treatment improves human quality, increasing mean sperm count, concentration, and motility [50]. The duration of the treatment is not well established. There is evidence for sperm parameters amelioration after one month of treatment [51], but longer period of treatment is also reported [49]. The dose of vitamin C must, however, is not excessive because high doses may act as a prooxidant, particularly in persons with the haptoglobin type 2-2 [52].

\section{Vitamin E}

Vitamin E (Tocopherol) is a fat-soluble organic compound mainly localized in cell membranes. It protects sperm cell membrane form oxidative stress-induced damage, preventing lipid peroxidation and capturing free hydroxyl radicals and superoxide [18]. Efforts have been made to establish if sperm parameters could be improved by vitamin E supplementation [53]. Its seminal plasma concentration significantly increases when it is administered at doses of 300 and $1200 \mathrm{mg} /$ day for three weeks [54]. The -tocopherol concentration in spermatozoa is independent from the total $\alpha$-tocopherol concentration in seminal plasma, and it significantly correlates with the percentage of motile spermatozoa [55]. A double-blind placebo-controlled trial showed an improved sperm function in vitro after $600 \mathrm{mg}$ daily administration of vitamin E for three months. The administration resulted in improved sperm motility and ability to bind the hamster oocyte in the hamster egg penetration test [53]. In other studies, lower doses (200 or 300mg/ day) have been used [56].

A placebo-controlled double-blind study reported an improvement of sperm motility in men with oligoasthenoteratozoospermia after vitamin $\mathrm{E}$ oral supplementation. The enhancement of sperm motility was associated with a decreased sperm production of MDA, the end product of the lipid peroxidation. Furthermore, in the course of the six-month treatment period, $21 \%$ of patients belonging to the treated group achieved pregnancy [57]. Seminal plasma MDA decrease seems to correlate with the percentage of successful attempts to achieve pregnancy. In a prospective study, 15 normozoospermic men with low fertilization rates in their earlier ART cycles were treated with $200 \mathrm{mg}$ /day of vitamin E for three months. The high MDA concentration declined to normal rate and the fertilization rate per cycle significantly improved after one month of treatment [56]. Finally, dietary habits seem also to play a role in semen quality, since a positive correlation has been found between vitamin E dietary intake and progressive and total motility 
[50]. This evidence suggests that vitamin E may have a positive effect on semen quality, enhancing the pregnancy rate. Altogether, these findings suggest that vitamin E could be a therapeutic choice for the treatment of male infertility. However, we would emphasize that, besides their interesting antioxidant properties, vitamin $\mathrm{E}$ analogs, especially tocopheryl succinate, can exert adverse effects on gap junctional intercellular communication, which could explain their controversial effects in spermatogenesis [52].

\section{Coenzyme Q10}

CoQ10 is the only lipid soluble antioxidant synthesized endogenously. In the Q-cycle it is present in three redox states: ubiquinone (CoQ10-oxidized), ubiquinol (CoQ10H2-reduced) and semiquinone (partially reduced, as radical). The reduced form has a higher antioxidant effect and concentration in the body is approximately $90 \%$ of total CoQ10. It inhibits protein and DNA oxidation and lipid peroxidation. CoQ10 regulates the mitochondrial electron transport in the respiratory chain, receiving electrons from complex I and complex II and passing them to complex III, and it transfers protons from fatty acids to the matrix. It also regulates the permeability transition pore opening and nutrition uptake through the voltage dependent anion channel (VDAC) of the outer mitochondrial membrane [17].

A number of clinical studies documented the beneficial effects of ubiquinone on male fertility. In fact, it has been shown to improve sperm parameters (concentration, motility, and morphology) in men with idiopathic OAT [58], with positive effects on the pregnancy rate [59]. It displays a protective effect against oxidative stress and sperm DNA damage. In addition, CoQ10 treatment improves sperm parameters and antioxidant status in infertile men with varicocele. Different therapeutic schemes are reported in literature. The lower dosage used is $90 \mathrm{mg}$ /day for a minimum of 3 months to a maximum of 9 months, but positive effects on sperm parameters have been obtained also by higher dosages (e.g., $100 \mathrm{mg}$ /day for three menses, $200 \mathrm{mg}$ for six months, etc.). The highest dosage reported is $300 \mathrm{mg}$ twice a day for twelve months [17].

Ubiquinol has a stronger antioxidant action in comparison with ubiquinone. It can regenerate other antioxidants such as vitamin $\mathrm{E}$ and vitamin $\mathrm{C}$. In addition, CoQ10 testicular biosynthesis is very active and high levels of ubiquinol are present in sperm [60]. Ubiquinol sperm concentration strongly correlates with sperm count, motility, and morphology. In addition, Q10-total concentration directly correlates with sperm motility [17]. Ubiquinol is administered orally at a dosage of 150 to $200 \mathrm{mg}$ daily, for at least four months.

Carnitine-ubiquinol combination displays a beneficial effect on sperm mitochondrial function of infertile men. It may be due to their uptake by VDAC of the outer mitochondrial membrane, along with the activity of carnitine palmitoyltransferase I (CPTI) in outer mitochondrial membrane. However, further studies are required to support this hypothesis [17].

\section{Carnitine}

Currently, carnitine is the molecule with antioxidant activity that has the greatest consensus in literature, especially in its forms L-carnitine and L-acetyl-carnitine. L-carnitine (mainly of exogenous origin though human beings are able to synthesize) is a high-polar, water-soluble quaternary amine. It acts as an essential cofactor for the transport of long chain fatty acids within the mitochondrial matrix in order to facilitate the oxidative processes and to enhance cellular energy production [61]. L-acetyl-carnitine, instead, is formed by the enzyme acetyl-L-carnitine transferase that modulates the intracellular and mitochondrial concentration of coenzyme A (CoA) and acetyl-CoA. Interestingly, high concentrations of carnitine are present in the male reproductive tract and particularly in the epididymis, suggesting its crucial role in energy metabolism and sperm maturation [62]. Hence, carnitine concentration in the ejaculate is considered a marker of epididymal function and some studies have shown a decreased L-carnitine concentration in the seminal fluid of patients with epididymitis [63].

The antioxidant properties of carnitine have been studied in men with male accessory gland inflammation. As previously said, inflammatory processes increase ROS production from leukocytes and/or spermatozoa with a consequent increased oxidative stress. Since, prostate-vesiculo-epididymitis (PVE) is the diagnostic category with a higher level of oxidative stress, some studies have evaluated the antioxidant properties of L-carnitine administration in patients with microbial PVE. The results of these studies have shown that the best effect is obtained administering first antibiotic and anti-inflammatory drug and subsequently L-carnitine. The co-administration of antimicrobial agents and antioxidants is less effective while treatment with L-carnitine alone has no effect [64].

The beneficial effects of L-carnitine on sperm parameters are well known. The data in the literature show a statistically significant improvement of sperm progressive motility in patients with OAT treated with L-carnitine or acetyl-L-carnitine at the dose of $3 \mathrm{~g} /$ day [65] for some months. Moreover, treatment with acetylL-carnitine increases sperm motility and viability in asymptomatic infertile patients with ROS overproduction and ultrasonographic evidence of PVE who already received antimicrobial therapy [62]. However, despite improving sperm parameters, there is no evidence of statistical significant variation of seminal plasma $\alpha$-glycosidase concentration (a marker of epididymal function) and of sperm membrane lipid peroxidation [66]. Combination therapy with L-carnitine $(2 \mathrm{~g} /$ day) and acetyl-L-carnitine $(500 \mathrm{mg}$ twice a day) is also effective in improving sperm quality in infertile patients (Lenzi et al., 2004) $[66,67]$ and it improves the total oxyradical scavenging capacity of the seminal fluid [68] and sperm parameters [68]. Interestingly, the addition of L-carnitine in samples to be cryopreservation improves significantly sperm quality [70].

In conclusion, the administration of carnitines is a rational and effective therapeutic strategy for the treatment of male infertility, since it leads to an improvement in sperm parameters. The best 
therapeutic scheme is represented by the co-administration of at least $2 \mathrm{~g}$ /daily of L-carnitine and at least $1 \mathrm{~g} /$ daily of acetyl-Lcarnitine for at least three months.

\section{Myoinositol}

Inositol is a component of the vitamin B complex. MYO, the most biologically important form in nature, is a precursor of second messengers and it is involved in several signal transduction mechanisms in the cell membrane. It regulates seminal plasma osmolarity, the expression of proteins essential for embryogenetic development and for sperm chemiotaxis and sperm motility. In addition, inositols are involved in sperm capacitation and acrosome reaction.

Incubation with MYO results in an increased sperm motility and in a higher number of spermatozoa retrieved by swim-up in both normozoospermic men and in patients with abnormal sperm parameters. This was associated with an improvement of sperm mitochondrial function in patients with OAT [71]. On this basis, the therapeutic use of MYO has been suggested in both in vivo and in vitro assisted reproductive techniques. Accordingly, oral supplementation with MYO seems to improve sperm parameters [72]. In particular, a double-blind, randomized, placebo-controlled study showed that patients with idiopathic infertility, treated for three months with MYO (2g twice daily), had a significant increase of sperm concentration, total count, progressive motility, and acrosome-reacted spermatozoa. In addition, MYO rebalances LH, FSH, and inhibin-B concentrations [73].

The most frequently reported supplementation strategy consists of a daily oral dose of $4 \mathrm{~g}$ (plus $400 \mu \mathrm{g}$ of folic acid), for at least two months.

\section{Lycopene}

Lycopene is a constituent of the human redox protection mechanism against ROS. Although few studies investigated its effects on sperm parameters, it seems to be a therapeutic choice in the treatment of idiopathic male infertility. In facts, its oral administration (2g twice a day for 3 months) improved sperm concentration and motility [74]. Furthermore, lycopene displays a protective effect against cryopreservation injury of post-thawing human spermatozoa. In fact, the addition of lycopene at a proper concentration to cryo-protectant reduces oxidative damage to sperm mitochondria in the freezing-thawing process, attenuates oxidative stress injury induced by ROS to sperm plasma membrane, and improves the anti-apoptosis sperm ability [75]. Various lycopene supplementation studies conducted on both humans and animals have shown promising results in alleviating male infertility by decreasing lipid peroxidation and DNA damage and increasing sperm count and viability. Improvement of these parameters indicates a reduction of the oxidative stress, and thus spermatozoa were less vulnerable to oxidative damage, which increases the chances of a normal sperm fertilizing the egg. Human trials have reported sperm parameter improvement and pregnancy rates with supplementation of 4-8mg of lycopene daily for 3-12 months. However, further detailed and extensive research is still required to determine the dosage and the usefulness of lycopene for male infertility treatment [76].

\section{Astaxanthin}

Astaxanthin is natural occurring xanthophyll. Only one study described its effects on human male fertility. A supplementation with $16 \mathrm{mg}$ daily of astaxanthin in 30 infertile patients resulted in higher sperm linear velocity, better capacity of binding the oocyte in the hamster penetration test, and higher total and per cycle pregnancy rates compared to the placebo group [77]. In a model of obese rats, vitamins $\mathrm{A}$ and $\mathrm{E}$ and astaxanthin administration was associated with better viability, motility, and morphology of spermatozoa obtained from the tail of the epididymis and with a significantly higher number of spermatogonium and Sertoli cells at the histological evaluation [78]. This finding led to the hypothesis that low sperm quality of obese men may be improved by the administration of this cocktail of antioxidants. However, other studies on a greater number of patients are needed to confirm if human sperm parameters may benefit from astaxanthin administration. Astaxanthin is administered in combined therapies at a daily dose of $16 \mathrm{mg}$. The duration of the treatment is not defined; in the mentioned study, astaxanthin was administered for three months [77].

\section{Serenoa repens}

Serenoa repens (saw palmetto) is a natural product with anti-inflammatory properties, derived from the American dwarf palm [79]. Currently, it is used to treat inflammatory symptoms in patients with benign prostatic hyperplasia and chronic prostatic inflammation [80]. Up to now, only a few and old studies have investigated its effects on sperm parameters. According to Ondrizek and colleagues, high concentration of Serenoa repens, Echinacea, or ginkgo inhibits human sperm motility. Moreover, Serenoa repens does not have any effect on oocyte penetration and sperm DNA integrity, while other herbs (such as Echinacea purpurea, Ginkgo biloba, and Hypericumperforatum) damage these parameters [81]. On this basis, Serenoa repens does not seem to ameliorate sperm parameters. Further studies are needed to confirm this conclusion.

\section{Micronutrients}

The concentration of micronutrients such as selenium, zinc and copper correlates with human sperm quality [18].

Selenium: Selenium is a micronutrient essential for normal testicular development, spermatogenesis, sperm motility and function. The lack of selenium has been correlated to seminiferous epithelium atrophy, disorders of spermatogenesis, maturation of spermatozoa in the epididymis, testis volume reduction, decreased sperm motility and altered sperm morphology (mainly in the head and in the midpiece) [82]. The exact mechanism by which selenium reduces oxidative stress and improves sperm parameters is still controversial. Its action seems to be mediated by seleno-enzymes, such as GPXs [83]. Selenium is given orally at a dosage that ranges from $(80$ to $300 \mu \mathrm{g})$ once a day, alone or in combination with antioxidants, for at least 3 months. 
Zinc: Zinc is a component of over 200 enzymes involved in the biosynthesis of nuclear acids, proteins and the process of cell division [18]. It has been reported to normalize oxido-sensitive indices and catalase-like activity in the seminal fluid of asthenozoospermic patients [84].

Zinc is given orally at the dose of $(220 \mathrm{mg})$ once or twice a day for 3 to 4 months, alone or in addition to folic acid (5mg daily) In combined therapies, it is administered at the dose of at least $10 \mathrm{mg}$ a day. It should be given in the esterified formulation as zinc picolinate that is best taken up by the intestinal tract [85]

Copper: Copper is a trace element whose seminal plasma concentration correlates with sperm quality [18]. A few studies report a positive effect of copper supplementation on sperm parameters in animals. In vitro supplementation with copper on ejaculated buffalo sperm led to a better protection through the process of dilution, equilibration and freeze-thawing at the dose of $32 \mu \mathrm{g} / \mathrm{L}$ compared to the dosage of $64 \mu \mathrm{g} / \mathrm{L}$ [86]. In addition, dietary copper supplementation at a dosage of $110 \mathrm{mg} / \mathrm{kg}$ has been shown to prevent the adverse effects produced by $12 \mathrm{mg} / \mathrm{kg} /$ day of tetrathiomolybdate in rat spermatozoa [87]. No studies have explored the effects of copper supplementation on human sperm parameters. Copper is not prescribed for the treatment of male infertility.

\section{Combined therapies}

The synergic effect of some component with different antioxidant properties has been studied. In a randomized trial, the daily administration of vitamin E (400mg) and selenium (225 $\mathrm{gg}$ ) for 3 months resulted in a significant decrease in MDA concentrations and an improvement of sperm motility [27]. In mice, the coadministration of vitamin C $(10 \mathrm{mg} / \mathrm{kg})$ and vitamin $\mathrm{E}(100 \mathrm{mg} / \mathrm{kg})$ led to a decrease of testicular MDA content, along with increased sperm count and decreased percentage of spermatozoa with abnormal form [44]. According to Greco and colleagues, the daily administration of vitamin C (1g) and vitamin E (1g) for 2 months significantly decreased the percentage of human DNA-fragmented spermatozoa. In addition, this association led to an improvement in the clinical pregnancy and implantation rates compared to the group of patients receiving placebo [88]. Furthermore, there is evidence for an increase of seminal vitamin E concentration and of its oxidative stability after cryopreservation if high amount of dietary -tocopheryl acetate is consumed. A decrease of seminal plasma ascorbate concentration is associated with a decreased fertilization rate. In rabbits, a high amount of dietary vitamin E (50 versus $200 \mathrm{mg} / \mathrm{kg}$ diet) significantly increased the level of vitamin $\mathrm{E}$ in the seminal fluid and the sperm oxidative stability after storage at $5^{\circ} \mathrm{C}$ for $24 \mathrm{~h}$.

Ascorbic acid showed a different effect in relation to the vitamin E status of animals: when associated with the higher level of vitamin $\mathrm{E}$ it increased vitamin $\mathrm{E}$ and the semen oxidative stability, whereas both parameters were reduced with lower vitamin E levels. Hence, their combination significantly improves the viability and kinetics of spermatozoa with an increased fertility rate [89]. These evidences sustain the role of the co-administration of vitamins $\mathrm{E}$ and $\mathrm{C}$ for the improvement of sperm quality in idiopathic male infertility.

The use of NAC, vitamin A, vitamin E and essential fatty acids in 27 infertile males increases sperm count and decreases ROS and 8-hydroxydeoxyguanine concentration in oligo-zoospermic patients. Moreover, the treatment improved the acrosome reaction rate and the proportion of PUFA in phospholipids and sperm membrane [85]. In addition, a 26-week-long daily administration of selenium $(200 \mu \mathrm{g})$ and NAC (600 mg) in 468 infertile men with idiopathic astheno-zoospermia resulted in an improvement of all sperm parameters. Furthermore, a positive relationship between seminal plasma concentration of NAC and selenium and sperm parameters has been found [90].

\section{other treatment}

Pine bark extract: The extract of the bark of the (Mediterranean) pine tree, Pinusmaritima, is rich in polyphenols, namely, anthocyanidins with antioxidant effect, and it reduces inflammatory reaction through the inhibition of the Cyclooxygenase enzymes (COX: 1 \& 2) [85] and of the Nuclear Factor Kappa B (NF$\mathrm{kB}$ ). Its potential use can be of interest in order to counteract sperm oxidative stress.

Lepidiummeyenii: Lepidiummeyenii, also called maca, is another plant belonging to the cruciferous (Brassica) family, growing in the Peruvian Andes Mountains. It has been considered phytoadaptogen that increases the production of the Heat Shock Protein ( $\mathrm{P}$ 72), by reducing the negative impact of stress on protein conformation and cell death [85]. Moreover, the potential increase of sexual desire induced by this plant without influencing testosterone concentration in adult men could be of importance for male infertility.

Polyunsaturated fatty acids: Polyunsaturated fatty acids have been recommended for nutrition thanks to their several benefits. In fact these fatty acids improve cell membrane fluidity and function, may possibly protect against cardiovascular diseases, and regarding male infertility may be potentially useful for the spermatogenesis [40].

\section{Summary}

The identification of etiopathogenetic mechanism(s) allows clinicians to select the optimal treatment to overcome male infertility. The therapeutic repertoire includes antibiotics, antiinflammatory drugs, antioxidants and micronutrients. Antibiotics are prescribed when a urogenital infection is identified. The most used antibiotics in clinical practice are quinolones (ciprofloxacin, levofloxacin, etc.), tetracyclines, macrolides, trimethoprim and $\beta$-lactam antibiotics (penicillin derivatives, cephalosporins, monobactams and carbapenems). Anti-inflammatory drugs should be given when leukocytospermia and/or inflammatory sign and/ or symptoms are present. Finally, antioxidants and micronutrients may be used to protect spermatozoa from oxidative stress overproduction that occurs in many clinical conditions. This class includes a vast array of molecules that may be prescribed alone or in combination. 
In conclusion, many non-hormonal compounds are available for the treatment of the infertile patient. This allows a customized therapeutic strategy.

\section{References}

1. Agarwal A, Ahmad G, Sharma R (2015) Reference values of reactive oxygen species in seminal ejaculates using chemiluminescence assay. Assist Reprod Genet 32(12): 1721-1729.

2. Agarwal A, Tvrda E, Sharma R (2014) Relationship amongst teratozoospermia, seminal oxidative stress and male infertility. Reprod BiolEndocrinol 12: 45.

3. Potts J, Pasqualotto F (2003) Seminal oxidative stress in patients with chronic prostatitis. Andrologia 35(5): 304-308.

4. Agarwal A, Virk G, Ong C, du Plessis S (2014) Effect of oxidative stress on male reproduction. World J Mens Health 32(1): 1-17.

5. Aitken R (1989) The role of free oxygen radicals and sperm function. Int J Androl 12(2): 95-97.

6. Hipler U, Gornig M, Hipler B, Romer W, Schreiber G (2000) Stimulation and scavestrogen-induced inhibition of reactive oxygen species generated by rat sertoli cells. Arch Androl 44(2): 147-154.

7. Ko E, Sabanegh E, Agarwal A (2014) Male infertility testing: reactive oxygen species and antioxidant capacity. Fertil Steril 102: 1518-1527.

8. Lavranos G, Balla M, Tzortzopoulou A, Syriou V, Angelopoulou R (2012) Investigating ROS sources in male infertility: a common end for numerous pathways. Reprod Toxicol 34(3): 298-307.

9. Saalu L (2010) The incriminating role of reactive oxygen species in idiopathic male infertility: an evidence based evaluation. Pak J BiolSci 13(9): 413-422.

10. Agarwal A, Hamada A, Esteves S (2012) Insight into oxidative stress in varicocele-associated male infertility: part 1. Nat Rev Urol 9(12): 678690.

11. Gharagozloo P, Aitken R (2011) The role of sperm oxidative stress in male infertility and the significance of oral antioxidant therapy. Hum Reprod 26(7): 1628-1640.

12. Chen S, Allam J, Duan Y, Haidl G (2013) Influence of reactive oxygen species on human sperm functions and fertilizing capacity including therapeutical approaches. Arch Gynecol Obstet 288(1): 191-199.

13. Agarwal A, Saleh R, Bedaiwy M (2003) Role of reactive oxygen species in the pathophysiology of human reproduction. FertilSteril 79(4): 829843.

14. Esteves S, Agarwal A (2011) Novel concepts in male infertility. IntBraz J Urol 37(1): 5-15.

15. Zini A, San Gabriel M, Baazeem A (2009) Antioxidants and sperm DNA damage: a clinical perspective. J Assist Reprod Genet 26: 427-432.

16. Thakur A, Littarru G, Funahashi I, Painkara U, Dange N, Chauhan $P$ (2015) Effect of ubiquinol therapy on sperm parameters and serum testosterone levels in oligoasthenozoospermic infertile men. J ClinDiagn Res 9(9): BC01-BC03.

17. Gvozdjakova A, Kucharska J, Dubravicky J, Mojto V, Singh R (2015) Coenzyme Q10, $\alpha$-tocopherol and oxidative stress could be important metabolic biomarkers of male infertility. Dis Markers 2015(2015).

18. Walczak-Jedrzejowska R, Wolski JK, Slowikowska-Hilczer J (2013) The role of oxidative stress and antioxidants in male fertility. Cen Euro J Urol 66(1): 60-67.

19. Fujii J, Iuchi Y, Matsuki S, Ishii T (2003) Cooperative function of antioxidant and redox systems against oxidative stress in male reproductive tissues. Asian J Androl 5(3): 231-242.

20. Peeker R, Abramsson L, Marklund SL (1997) Superoxide dismutase isoenzymes in human seminal plasma and spermatozoa. Mol Hum Reprod 3(12): 1061-1066.

21. Kumagai A, Kodama H, Kumagai J, Fukuda J, Kawamura K, et al. (2002) Xanthine oxidase inhibitors suppress testicular germ cell apoptosis induced by experimental cryptorchidism. Mol Hum Reprod 8(2): 118123.

22. Hosen MB, Islam MR, Begum F, Kabir Y, Howlader MZH (2015) Oxidative stress induced sperm DNA damage, a possible reason for male infertility. Iranian Journal of Reproductive Medicine 13(9): 525-532.

23. Forouzanfar M, Abid A, Hosseini SM, Hajian M, Nasr Esfahani MH (2013) Supplementation of sperm cryopreservation media with cell permeable superoxide dismutase mimetic agent (MnTE) improves goat blastocyst formation. Cryobiology 67(3): 394-397.

24. Shafiei M, Forouzanfar M, Hosseini SM, Esfahani MHN (2015) The effect of superoxide dismutase mimetic and catalase on the quality of postthawed goat semen. Theriogenology 83(8): 1321-1327.

25. Kobayashi T, Miyazaki T, Natori M, Nozawa S (1991) Protective role of superoxide dismutase in human sperm motility: superoxide dismutase activity and lipid peroxide in human seminal plasma and spermatozoa. Hum Reprod 6(7): 987-991.

26. Sabouhi S, Salehi Z, Bahadori MH, Mahdavi M (2015) Human catalase gene polymorphism (CAT C-262T) and risk of male infertility. Andrologia 47(1): 97-101.

27. Makker A, Agarwal A, Sharma R (2009) Oxidative stress \& male infertility. Ind J Med Res 129(4): 357-367.

28. Galecka E, Jacewicz R, Mrowicka M, Florkowski A, Galecki P (2008) Antioxidative enzymes-structure, properties, functions. PolskiMerkuriuszLekarski 25(147): 266-268.

29. Diaconu M, Tangat Y, Böhm D (2006) Failure of phospholipid hydroperoxide glutathione peroxidase expression in oligoasthenozoospermia and mutations in the PHGPx gene. Andrologia 38(4): 152-157.

30. Tripodi L, Tripodi A, Mammí C, Pullé C, Cremonesi F (2003) Pharmacological action and therapeutic effects of glutathione on hypokinetic spermatozoa for enzymatic-dependent pathologies and correlated genetic aspects. Clin Exp Obstet Gynecol 30(2-3): 130-136.

31. Lenzi A, Lombardo F, Sgrò P (2003) Use of carnitine therapy in selected cases of male factor infertility: a double-blind crossover trial. Fertil Steril 79(2): 292-300.

32. Irvine DS (1996) Glutathione as a treatment for male infertility. Rev Reprod 1(1): 6-12.

33. Opuwari CS, Henkel RR (2016) An update on oxidative damage to spermatozoa and oocytes. BioMed Res Int.

34. da Silva RF, dos Santos Borges C, e Silva PV (2016) The coadministration of $\mathrm{N}$-acetylcysteine ameliorates the effects of arsenic trioxide on the male mouse genital system. Oxidative Medicine and Cellular Longevity.

35. Ciftci H, Verit A, Savas M, Yeni E, Erel O (2009) Effects of N-acetylcysteine on semen parameters and oxidative/antioxidant status. Urology 74(1): 73-76.

36. Cheraghi E, Mehranjani MS, Shariatzadeh MA, Esfahani MHN, Ebrahimi Z (2016) N-Acetylcysteine improves oocyte and embryo quality in polycystic ovary syndrome patients undergoing intracytoplasmic sperm injection: an alternative to metformin. Reprod, Fertil Dev 28(6): 723-731.

37. Livera G, Rouiller-Fabre V, Durand P, Habert R (2000) Multiple effects of retinoids on the development of Sertoli, germ, and Leydig cells of fetal and neonatal rat testis in culture. Biol Reprod 62(5): 1303-1314.

38. Maya-Soriano MJ, Taberner E, Sabés-Alsina M, López-Béjar M (2013) Retinol might stabilize sperm acrosomal membrane in situations of oxidative stress because of high temperatures, Theriogenology 79(2): 367-373.

39. Al-Azemi MK, Omu AE, Fatinikun T, Mannazhath N, Abraham S (2009) Factors contributing to gender differences in serum retinol and $\alpha$-tocopherol in infertile couples. Reprod BioMed Online 19(4): 583590.

40. Comhaire F, Mahmoud A (2016) The andrologist's contribution to a better life for ageing men: part 2. Andrologia 48(1): 99-110. 
41. Jacob RA, Pianalto FS, Agee RE (1992) Cellular ascorbate depletion in healthy men. J Nutri 122(5): 1111-1118.

42. Frei B, England L, Ames BN (1989) Ascorbate is an outstanding antioxidant in human blood plasma. Proc Nati Acad Sci U S A 86(16): 6377-6381.

43. Doba T, Burton GW, Ingold KU (1985) Antioxidant and co-antioxidant activity of vitamin $C$. The effect of vitamin $C$, either alone or in the presence of vitamin $\mathrm{E}$ or a water-soluble vitamin $\mathrm{E}$ analogue, upon the peroxidation of aqueous multilamellar phospholipid liposomes. Biochimica et BiophysicaActa 835(2): 298-303.

44. Mishra M, Acharya UR (2004) Protective action of vitamins on the spermatogenesis in lead-treated Swiss mice. J Trace Elem Med Biol 18(2): 173-178.

45. Thiele JJ, Freisleben HJ, Fuchs J, Ochsendorf FR (1995) Ascorbic acid and urate in human seminal plasma: determination and interrelationships with chemiluminescence in washed semen. Hum Reprod 10(1): 110115.

46. Song GJ, Norkus EP, Lewis V (2006) Relationship between seminal ascorbic acid and sperm DNA integrity in infertile men. Int J Androl 29(6): 569-575

47. Lanzafame FM, La Vignera S, Vicari E, Calogero AE (2009) Oxidative stress and medical antioxidant treatment in male infertility. Reproductive BioMed Online 19(5): 638-659.

48. Wen Y, Cooke T, Feely J (1997) The effect of pharmacological supplementation with vitamin $\mathrm{C}$ on low-density lipoprotein oxidation Br J Clin Pharmacol 44(1): 94-97.

49. Levine M, Conry-Cantilena C, Wang Y (1996) Vitamin C pharmacokinetics in healthy volunteers: evidence for a recommended dietary allowance. Proc Nat Acad Sci U S A 93(8): 3704-3709.

50. Eskenazi B, Kidd SA, Marks AR, Sloter E, Block G, et al. (2005) Antioxidant intake is associated with semen quality in healthy men. Hum Reprod 20(4): 1006-1012.

51. Dawson EB, Harris WA, Teter MC, Powell LC (1992) Effect of ascorbic acid supplementation on the sperm quality of smokers. Fertil Steril 58(5): 1034-1039.

52. Comhaire F (2010) The role of food supplementation in the treatment of the infertile couple and for assisted reproduction. Andrologia 42(5): 331-340.

53. Kessopoulou E, Powers EJ, Sharma KK (1995) A double-blind randomized placebo cross-over controlled trial using the antioxidant vitamin $\mathrm{E}$ to treat reactive oxygen species associated male infertility. Fertil Steril 64(4): 825-831.

54. Moilanen J, Hovatta O (1995) Excretion of alpha-tocopherol into human seminal plasma after oral administration. Andrologia 27(3): 133-136.

55. Thérond P, Auger J, Legrand A, Jouannet P (1996) $\alpha$-tocopherol in human spermatozoa and seminal plasma: relationships with motility, antioxidant enzymes and leukocytes. Mol Hum Reprod 2(10): 739-744.

56. Geva E, Bartoov B, Zabludovsky N, Lessing JB, Lerner-Geva L, et al. (1996) The effect of antioxidant treatment on human spermatozoa and fertilization rate in an in vitro fertilization program. Fertility and Sterility 66(3): 430-434.

57. Suleiman SA, Ali ME, Zaki ZMS, El-Malik EMA, Nasr MA (1996) Lipid peroxidation and human sperm motility: protective role of vitamin E. J Androl 17(5): 530-537.

58. Balercia B, Buldreghini E, Vignini A (2009) Coenzyme Q10 treatment in infertile men with idiopathic asthenozoospermia: a placebo-controlled, double-blind randomized trial. Fertil Steril 91(5): 1785-1792.

59. Safarinejad MR (2012) The effect of coenzyme Q 10 supplementation on partner pregnancy rate in infertile men with idiopathic oligoasthenoteratozoospermia: an open-label prospective study." Int Urol Nephrol 44(3): 689-700.

60. Mancini G, Conte D, Milardi L, de Marinis L, Littarru GP (1998) Relationship between sperm cell ubiquinone and seminal parameters in subjects with and without varicocele. Andrologia 30(1): 1-4.
61. Agarwal A, Said TM (2004) Carnitines and male infertility. Reprod BioMed Online 8(4): 376-384.

62. Vicari E, Calogero AE (2001) Effects of treatment with carnitines in infertile patients with prostato-vesiculo-epididymitis. Hum Reprod 16(11): 2338-2342.

63. CooperTG, Weidner W, Nieschlag E (1990) The influence of inflammation of the human male genital tract on secretion of the seminal markers $\alpha$ glucosidase, glycerophosphocholine, carnitine, fructose and citric acid. Int J Androl 13(5): 329-336.

64. Vicari E, Rubino C, De Palma A (2001) Antioxidant therapeutic efficiency after the use of carnitine in infertile patients with bacterial or non-bacterial prostato-vesiculo-epididymitis. Arch Ital Urol Androl 73(1): 15-25.

65. Vitali G, Parente R, Melotti C (1995) Carnitine supplementation in human idiopathic asthenospermia: clinical results. Drugs Exp Clin Res 21(4): 157-159.

66. Lenzi A, Sgrò P, Salacone P (2004) A placebo-controlled double-blind randomized trial of the use of combined L-carnitine and L-acetylcarnitine treatment in men with asthenozoospermia. Fertil Steril 8(16): 1578-1584.

67. Lenzi A, Picardo M, Gandini L (1994) Glutathione treatment of dyspermia: effect on the lipoperoxidation process. Hum Reprod 9(11): 2044-2050.

68. Balercia G, Regoli F, Armeni T, Koverech A, Mantero F, etal. (2005) Placebocontrolled double-blind randomized trial on the use of l-carnitine, l-acetylcarnitine, or combined l-carnitine and l-acetylcarnitine in men with idiopathic asthenozoospermia. Fertil Steril 84(3): 662-671.

69. De Rosa M, Boggia B, Amalfi B (2005) Correlation between seminal carnitine and functional spermatozoal characteristics in men with semen dysfunction of various origins. Drugs R D 6(1): 1-9.

70. Banihani S, Agarwal A, Sharma R, Bayachou M (2014) Cryoprotective effect of l-carnitine on motility, vitality and DNA oxidation of human spermatozoa. Andrologia 46(6): 637-641.

71. Condorelli RA, La Vignera S, Bellanca S, Vicari E, Calogero AE (2012) Myoinositol: does it improve sperm mitochondrial function and sperm motility? Urology 79(6): 1290-1295.

72. Gulino FA, Leonardi E, Marilli I (2016) Effect of treatment with myoinositol on semen parameters of patients undergoing an IVF cycle: in vivo study. Gynecol Endocrinol 32(1): 65-68.

73. Calogero E, Gullo G, La Vignera S, Condorelli RA, Vaiarelli A (2015) Myoinositol improves sperm parameters and serum reproductive hormones in patients with idiopathic infertility: a prospective doubleblind randomized placebo-controlled study. Andrology 3(3): 491-495.

74. Gupta NP, Kumar R (2002) Lycopene therapy in idiopathic male infertility-a preliminary report. Int Urol Nephrol 34(3): 369-372.

75. Liang ZW, Guo KM, Dai XF (2015) Protective effect of lycopene on human spermatozoa during cryopreservation and its mechanism. Zhonghua Nan Ke Xue 21(6): 521-526.

76. Durairajanayagam D, Agarwal A, Ong C, Prashast P (2014) Lycopene and male infertility. Asian J Androl 16(3): 420-425.

77. Comhaire FH, El Garem Y, Mahmoud A, Eertmans F, Schoonjans F (2005) Combined conventional/antioxidant. Asian J Androl 7(3): 257-262.

78. Mortazavi M, Salehi I, Alizadeh Z, Vahabian M, Roushandeh AM (2014) Protective effects of antioxidants on sperm parameters and seminiferous tubules epithelium in high fat-fed rats," J Reprod Infertil 15(1): 22-28

79. MacDonald R, Tacklind RW, Rutks I, Wilt TJ (2012) Serenoa repens monotherapy for benign prostatic hyperplasia (BPH): an updated Cochrane systematic review. BJU Int 109(12): 1756-1761.

80. Latil MT, Pétrissans MT, Rouquet J, Robert G, de la Taille A (2015) Effects of hexanic extract of Serenoa repens (Permixon® $160 \mathrm{mg}$ ) on inflammation biomarkers in the treatment of lower urinary tract symptoms related to benign prostatic hyperplasia. Prostate $75(16)$ 1857-1867. 
81. Ondrizek RR, Chan PJ, Patton WC, King A (1999) Inhibition of human sperm motility by specific herbs used in alternative medicine. J Assist Reprod Gen 16(2): 87-91.

82. Atig F, Raffa M, Ali MB, Abdelhamid K, Saad A, Ajina M (2011) Altered antioxidant status and increased lipid per-oxidation in seminal plasma of Tunisian infertile men. Int J Biol Sci 8(1): 139-149.

83. Agarwal A, Sekhon LH (2011) Oxidative stress and antioxidants for idiopathic oligo-astheno-terato-spermia: is it justified. Indian J Urol 27(1): 74-85.

84. Hadwan MH, Almashhedy LA, Alsalman ARS (2015) Oral zinc supplementation restores superoxide radical scavengers to normal levels in spermatozoa of Iraqi asthenospermic patients. Int J Vitam Nutr Res 85(3-4): 165-173.

85. Comhaire FH, Christophe AB, Zalata AA, Dhooge WS, Mahmoud AMA, Depuydt CE (2000) The effects of combined conventional treatment, oral antioxidants and essential fatty acids on sperm biology in subfertile men. Prostaglandins Leukotrienes and Essential Fatty Acids 63(3): 159165.
86. Tabassomi M, Alavi-Shoushtari SM (2013) Effects of in vitro copper sulphate supplementation on the ejaculated sperm characteristics in water buffaloes (Bubalusbubalis). Vet Res Forum 4(1): 31-36.

87. Lyubimov V, Smith JA, Rousselle SD (2004) The effects of tetrathiomolybdate (TTM, NSC-714598) and copper supplementation on fertility and early embryonic development in rats. Reprod Toxicol 19(2): 223-233.

88. Greco E, Iacobelli M, Rienzi L, Ubaldi F, Ferrero S, et al. (2005) Reduction of the incidence of sperm DNA fragmentation by oral antioxidant treatment. J Androl 26(3): 349-353.

89. Castellini P, Lattaioli M, Bernardini M, Dal Bosco A (2000) Effect of dietary $\alpha$-tocopheryl acetate and ascorbic acid on rabbit semen stored at $5{ }^{\circ} \mathrm{C}$. Theriogenology 54(4): 523-533.

90. Safarinejad MR, Safarinejad S (2009) Efficacy of selenium and/or $\mathrm{N}$-acetyl-cysteine for improving semen parameters in infertile men: a double-blind, placebo controlled, randomized study. J Urol 181(2): 741751. 